\title{
Stratified Necessary Conditions for Differential Inclusions with State Constraints
}

\author{
Piernicola Bettiol, Andrea Boccia ${ }^{\dagger}$ and Richard B. Vinter ${ }^{\dagger}$
}

January 27, 2014

\begin{abstract}
The concept of stratified necessary conditions for optimal control problems, whose dynamic constraint is formulated as a differential inclusion, was introduced by F. H. Clarke. These are conditions satisfied by a feasible state trajectory that achieves the minimum value of the cost over state trajectories whose velocities lie in a time-varying open ball of specified radius about the velocity of the state trajectory of interest. Considering different radius functions stratifies the interpretation of 'minimizer'. In this paper we prove stratified necessary conditions for optimal control problems involving pathwise state constraints. As was shown by Clarke in the state constraint-free case, we find that, also in our more general setting, the stratified necessary conditions yield generalizations of earlier optimality conditions for unbounded differential inclusions as simple corollaries. Some examples are provided, giving insights into the nature of the hypotheses invoked for the derivation of stratified necessary conditions and into the scope for their further refinement.
\end{abstract}

Keywords: Necessary Conditions, Optimal Control, Differential Inclusions, State Constraints.

\section{Introduction}

This paper provides necessary conditions of optimality for state constrained optimal control problems ${ }^{1}$ in which the dynamic constraint is modelled as a differential inclusion:

$$
(P) \quad\left\{\begin{array}{l}
\text { Minimize } \ell(x(S), x(T)) \\
\text { over } \operatorname{arcs} x(.) \in W^{1,1}\left([S, T] ; \mathbb{R}^{n}\right) \text { satisfying } \\
\dot{x}(t) \in F(t, x(t)), \text { for a.e. } t \in[S, T] \\
(x(S), x(T)) \in E \\
h(t, x(t)) \leq 0, \text { for all } t \in[S, T]
\end{array}\right.
$$

\footnotetext{
* Laboratoire de Mathematiques, Université de Bretagne Occidentale, 6 Avenue Victor Le Gorgeu, 29200 Brest, France, e-mail: piernicola.bettiol@univ-brest.fr

${ }^{\dagger}$ Department of Electrical and Electronic Engineering, Imperial College London, Exhibition Road, London, SW7 2BT, UK, e-mail: a.boccia@imperial.ac.uk, r.vinter@imperial.ac.uk

${ }^{1}$ The main results of this paper were announced, without detailed proofs, in [2].
} 
The data for $(P)$ comprises an interval $[S, T] \subset \mathbb{R}(T>S)$, a set $E \subset \mathbb{R}^{n} \times \mathbb{R}^{n}$, functions $\ell: \mathbb{R}^{n} \times \mathbb{R}^{n} \rightarrow \mathbb{R}$ and $h:[S, T] \times \mathbb{R}^{n} \rightarrow \mathbb{R}$, and a set-valued map $F:[S, T] \times \mathbb{R}^{n} \leadsto \mathbb{R}^{n}$.

Absolutely continuous $\operatorname{arcs} x($.$) that satisfy the differential inclusion \dot{x}(t) \in F(t, x(t))$ a.e. are called $F$-trajectories. If additionally they satisfy all the other constraints in $(P)$, they are referred to as feasible F-trajectories. Feasible $F$-trajectories that minimize the cost over the set of feasible $F$-trajectories are called minimizers.

Suppose for the time being that the state constraint $h(t, x(t)) \leq 0$ is absent from the above formulation. (This case is captured by setting $h(.,.) \equiv-1$.) The earliest general necessary conditions for an $F$-trajectory $\bar{x}($.$) to be a minimizer were$ provided by F. H. Clarke, under hypotheses that included the requirement that the multifunction $F(.,$.$) is convex valued and bounded and that F(t,$.$) is Lipschitz con-$ tinuous w.r.t. the $x$ variable, in the following sense: there exists $\epsilon>0$ and $k(.) \in L^{1}$ such that

$$
F(t, x) \subset F\left(t, x^{\prime}\right)+k(t)\left|x-x^{\prime}\right| \overline{\mathbb{B}} \quad \text { for all } x, x^{\prime} \in \bar{x}(t)+\epsilon \overline{\mathbb{B}} \text {, a.e.. }
$$

The necessary conditions assert that there exists an absolutely continuous function $p($.$) called the co-state function which, together with the minimizing F$-trajectory $\bar{x}($.$) , satisfies a generalization of Hamilton's system of equations (the 'Hamiltonian$ inclusion'), and a set of boundary conditions (the 'transversality conditions'). Subsequent work on such conditions (see [10], [9] and, for an expository account, [12]) culminated in a set of conditions valid for unbounded, possibly non-convex valued differential inclusions, in which the generalized Hamiltonian inclusion was replaced by a combination of the Generalized Euler Lagrange inclusion

$$
\dot{p}(t) \in \operatorname{co}\left\{q \mid(q, p(t)) \in N_{G r F(t, .)}(\bar{x}(t), \dot{\bar{x}}(t))\right\} \text { a.e. }
$$

and the Weierstrass condition

$$
p(t) \cdot \dot{\bar{x}}(t) \geq p(t) \cdot v \quad \text { for all } v \in F(t, \bar{x}(t)), \text { a.e.. }
$$

(The notation $N_{G r F(t, .)}$ will be explained shortly.) Also, the Lipschitz continuity hypothesis on $F(t,).(1.1)$ was replaced by a less restrictive condition, a typical example of which is: there exist $k(.) \in L^{1}, \epsilon>0$ and $\beta \geq 0$ such that, for all $N \geq 0$,

$$
\begin{array}{r}
F(t, x) \cap(\dot{\bar{x}}(t)+N \overline{\mathbb{B}}) \subset F\left(t, x^{\prime}\right)+(k(t)+\beta N)\left|x-x^{\prime}\right| \overline{\mathbb{B}} \\
\text { for all } x, x^{\prime} \in \bar{x}(t)+\epsilon \overline{\mathbb{B}}, \text { a.e.. }
\end{array}
$$

(Notice that the Lipschitz constant grows linearly with the size of the intersecting ball on the left side.) The motivation here is that, for unbounded differential inclusions, the earlier imposed Lipschitz continuity condition (1.1) is overly restrictive. If, for example, we wish to cover differential inclusions representing a two state differential inequality of the form

$$
\dot{x}_{1} \leq x_{1} \dot{x}_{2}
$$


the Lipschitz continuity is not satisfied because the Hausdorff distance between the sets $\left\{\left(e_{1}, e_{2}\right) \mid e_{1} \leq x_{1} e_{2}\right\}$ and $\left\{\left(e_{1}, e_{2}\right) \mid e_{1} \leq x_{1}^{\prime} e_{2}\right\}$ is infinite, for $x_{1} \neq x_{1}^{\prime}$. On the other hand, condition (1.4), which we call the 'pseudo-Lipschitz' continuity condition with linear growth, is clearly satisfied.

More recently, Clarke has taken this research in a new direction, by introducing the concept of 'stratified' necessary conditions, w.r.t. a given radius function $r(t)$ or, more generally, a radius multifunction. Here, the object is to derive necessary conditions of optimality when the feasible $F$-trajectory $\bar{x}($.$) is a minimizer in com-$ parison to all feasible $F$-trajectories $x($.$) whose velocities are restricted to satisfy the$ condition:

$$
\dot{x}(t) \in \dot{\bar{x}}(t)+r(t) \mathbb{B} .
$$

Clarke showed that if the data satisfies a form of the pseudo-Lipschitz condition (1.4), now incorporating the radius function $r($.$) , namely: there exist \epsilon>0$ and $k(.) \in L^{1}$ such that

$F(t, x) \cap(\dot{\bar{x}}(t)+r(t) \mathbb{B}) \subset F\left(t, x^{\prime}\right)+k(t)\left|x-x^{\prime}\right| \overline{\mathbb{B}} \quad$ for all $x, x^{\prime} \in \bar{x}(t)+\epsilon \overline{\mathbb{B}}$, a.e.,

and also satisfies a technical condition, referred to as the 'tempered growth' condition, then the generalized Euler Lagrange inclusion (1.2) and a restricted form of the Weierstrass condition (1.3) are satisfied (for some $p($.$) ), that is$

$$
p(t) \cdot \dot{\bar{x}}(t) \geq p(t) \cdot v \quad \text { for all } v \in F(t, \bar{x}(t)) \cap(\dot{\bar{x}}(t)+r(t) \mathbb{B}) \text {, a.e.. }
$$

The stratified necessary conditions have a number of useful consequences:

First, they yield as straightforward corollaries necessary conditions under refinements of the pseudo-Lipschitz condition with 'linear growth' hypothesis (1.4); these are obtained by considering a sequence of radius functions tending to infinity, a.e. We discuss several of these below. Some such refinements could, possibly, be obtained by techniques similar to those originally used to prove necessary conditions under hypothesis (1.4), but only at the price of taking apart and reworking a rather complicated analysis.

Second, they are the starting point for the derivation of state-of-the-art necessary conditions for problems with 'mixed' (control and state) constraints [8], [7]. This is because, if the controlled differential inclusion and the mixed constraint is recast as a differential inclusion, the differential inclusion satisfies the hypotheses for application of the stratified necessary conditions, under very general hypotheses on the original data.

Third they provide a framework for studying necessary conditions for a weak minimizer $\bar{x}($.$) , i.e. an admissible arc that, for some \delta>0$, is minimizing w.r.t. admissible arcs that satisfy

$$
|x(t)-\bar{x}(t)|+|\dot{x}(t)-\dot{\bar{x}}(t)|<\delta \quad \text { a.e.. }
$$

For problems in the calculus of variations in which no dynamic constraints are imposed on feasible trajectories, it can be shown, via straightforward application of 
the Pontryagin maximum principle or direct variational analysis, that weak minimizers (in the above sense) satisfy the Euler Lagrange, transversality conditions and a restricted form of the Weierstrass condition. If, however, we now add a dynamic constraint in the form of a differential inclusion $\dot{x} \in F(t, x)$, weak minimizers may fail to satisfy the above necessary conditions, even in seemingly benign situations, in which $F(t,$.$) is convex valued and satisfies the global Lipschitz continuity condition$ (1.1). The stratified theory permits us to explore the 'extra' hypotheses required to exclude this surprising phenomenon.

The principal contribution of this paper is to show that the stratified necessary conditions remain valid (with appropriate modifications), when a pathwise state constraint, $h(t, x(t)) \leq 0$, is included in the problem formulation $(\mathrm{P})$. The conditions obtained reduce precisely to those in [3] and [4] when the state constraint is omitted. We allow general (set valued) radius multifunctions $R($.$) in place of$ $\dot{\bar{x}}()+.r(.) \mathbb{B}$, as in [4]. We also examine examples aimed at providing insights into the ultimate limitations on possible generalizations of such necessary conditions.

Finally, we comment on the methodology for proving stratified necessary conditions. It might be thought that this was a simple matter of replacing (for each number $N>0)$ the possibly unbounded multifunction $F(.,$.$) by the bounded multifunction$ $F(t,.) \cap(\dot{\bar{x}}(t)+(r(t) \wedge N) \overline{\mathbb{B}})$, applying known necessary conditions, and passage to the limit, as $N \rightarrow \infty$. But this would require $F(t,.) \cap(\dot{\bar{x}}(t)+(r(t) \wedge N) \overline{\mathbb{B}})$ to have Lipschitz continuity properties; unfortunately, however, the constructed multifunction can fail to be continuous (let alone Lipschitz continuous in some useful sense), even if $F(t,$.$) satisfies the (global) Lipschitz condition (1.1). We follow a simple and$ effective proof technique proposed by Clarke ('lifting'), now in the state-constrained setting, in which we deal not directly with $F(t,.) \cap(\dot{\bar{x}}(t)+(r(t) \wedge N) \overline{\mathbb{B}})$ itself, but instead through a proxy, a related multifunction taking as values sets in a higher dimensional state space, which has much better regularity properties.

The following notation will be used throughout the paper. For vectors $x \in \mathbb{R}^{n}$, $|x|$ denotes the Euclidean length. $\mathbb{B}$ denotes the open unit ball in $\mathbb{R}^{n}$. It's closure is written $\overline{\mathbb{B}}$. Given a multi-function $\Gamma():. \mathbb{R}^{n} \leadsto \mathbb{R}^{k}, \operatorname{Gr} \Gamma($.$) is the set \{(x, v) \in$ $\left.\mathbb{R}^{n} \times \mathbb{R}^{k} \mid v \in \Gamma(x)\right\}$. Give a set $A \subset \mathbb{R}^{n}$ and a point $x \in \mathbb{R}^{n}$, we denote by $d_{A}(x)$ the Euclidean distance of a point $x \in \mathbb{R}^{n}$ from $A$ :

$$
d_{A}(x):=\inf \{|x-y| \mid y \in A\} .
$$

$W^{1,1}\left([S, T] ; \mathbb{R}^{n}\right)$ is the space of absolutely continuous $\mathbb{R}^{n}$-valued function on $[S, T]$ with the norm

$$
\|x\|_{W 1,1}:=|x(S)|+\int_{S}^{T}|\dot{x}(t)| d t .
$$

We write $W^{1,1}$ in place of $W^{1,1}\left([S, T] ; \mathbb{R}^{n}\right)$ when the meaning is clear. We denote by $N B V^{+}[S, T]$ the space of increasing, real-valued functions $\mu($.$) on [S, T]$ of bounded variation, vanishing at the point $S$ and right continuous on $(S, T)$. The total variation of a function $\mu(.) \in N B V^{+}[S, T]$ is written $\|\mu\|_{T . V .}$. As is well known, each 
function $\mu(.) \in N B V^{+}[S, T]$ defines a Borel measure on $[S, T]$. This associated measure is also denoted $\mu$.

We shall use several constructs of nonsmooth analysis. Given a closed set $D \subset \mathbb{R}^{k}$ and a point $\bar{x} \in D$, the normal cone $N_{D}(\bar{x})$ of $D$ at $\bar{x}$ is defined to be

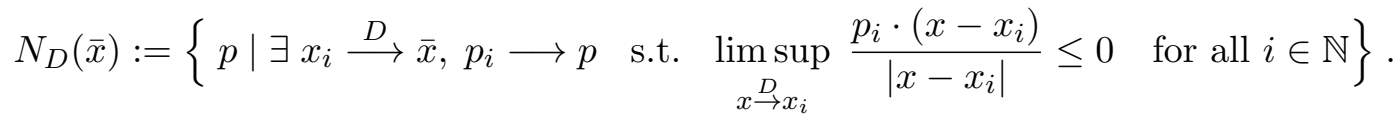

Here the notation $x_{i} \stackrel{D}{\longrightarrow} \bar{x}$ indicates that $x_{i} \in D$ along the convergent sequence $x_{i} \rightarrow \bar{x}$.

Given a lower semicontinuous function $f: \mathbb{R}^{k} \rightarrow \mathbb{R} \cup\{+\infty\}$ and a point $\bar{x} \in$ $\operatorname{dom} f:=\left\{x \in \mathbb{R}^{k} \mid f(x)<+\infty\right\}$, the subdifferential of $f$ at $\bar{x}$ (termed the 'limiting subdifferential' in [6]) is the set

$$
\begin{aligned}
\partial f(\bar{x}):= & \left\{\xi \mid \exists \xi_{i} \rightarrow \xi \text { and } x_{i} \stackrel{\operatorname{dom} f}{\longrightarrow} \bar{x}\right. \text { such that } \\
& \left.\limsup _{x \rightarrow x_{i}} \frac{\xi_{i} \cdot\left(x-x_{i}\right)-f(x)+f\left(x_{i}\right)}{\left|x-x_{i}\right|} \leq 0 \text { for all } i \in \mathbb{N}\right\} .
\end{aligned}
$$

For details of definitions and properties of these and related objects, we refer the reader to $[5],[6],[11]$ and $[12]$.

\section{Stratified Necessary Conditions for State Constrained Optimal Control Problems}

In this section we provide necessary conditions for a feasible $F$-trajectory $\bar{x}($.$) to$ be a minimizer w.r.t. a given radius multi-function. Some direct implications of these conditions are then stated in the form of corollaries. Proofs will follow in later sections.

Take a feasible $F$-trajectory $\bar{x}($.$) for (P)$. We shall say that $R($.$) is a radius multi-$ function (for $\bar{x}()$.$) if R($.$) is a measurable multifunction and, for each t \in[S, T], R(t)$ is an open convex set such that

$$
\dot{\bar{x}}(t) \in R(t) \quad \text { a.e. }
$$

Definition. $\bar{x}($.$) is a W^{1,1}$ local minimizer w.r.t. a given radius multi-function $R($. for $\bar{x}($.$) , if there exists \epsilon>0$ such that

$$
\ell(x(S), x(T)) \geq \ell(\bar{x}(S), \bar{x}(T))
$$

for all feasible $F$-trajectories $x($.$) such that \|x-\bar{x}\|_{W^{1,1}} \leq \epsilon$ and

$$
\dot{x}(t) \in R(t) \quad \text { a.e.. }
$$

The following hypotheses are invoked: for some $\epsilon>0$, 
(H1) $\ell(.,$.$) is Lipschitz continuous on a neighborhood of (\bar{x}(S), \bar{x}(T))$ and $E \subset \mathbb{R}^{n} \times$ $\mathbb{R}^{n}$ is a closed set.

(H2) $h(.,$.$) is upper semicontinuous near (t, \bar{x}(t))$, for all $t \in[S, T]$, and there exists a constant $k_{h}$ such that

$$
\left|h\left(t, x^{\prime}\right)-h(t, x)\right| \leq k_{h}\left|x^{\prime}-x\right|,
$$

for all $t$ in $[S, T]$ and all $x^{\prime}, x \in \bar{x}(t)+\epsilon \overline{\mathbb{B}}$.

(H3) $F(.,$.$) takes values non-empty subset of \mathbb{R}^{n}$. The restriction of $F(t,$.$) to \bar{x}(t)+$ $\epsilon \overline{\mathbb{B}}$ has closed graph. $F(.,$.$) is measurable w.r.t. the product \mathcal{L} \times \mathcal{B}$-algebra, where $\mathcal{L}$ and $\mathcal{B}$ denote the $\sigma$-algebras of Lebesgue subsets of $[S, T]$ and of Borel subsets of $\mathbb{R}^{n}$ respectively.

(H4) There exists a function $k(.) \in L^{1}(S, T)$ such that

$$
F\left(t, x^{\prime}\right) \cap R(t) \subset F(t, x)+k(t)\left|x-x^{\prime}\right| \overline{\mathbb{B}},
$$

for all $x, x^{\prime} \in \bar{x}(t)+\epsilon \overline{\mathbb{B}}$, a.e. $t \in[S, T]$.

(H5) There exist numbers $r_{0}>0$ and $\gamma \in(0,1)$, and a function $r(.) \in L^{1}(S, T)$ such that $r(t) \geq r_{0}$ a.e. and
i) $\dot{\bar{x}}(t)+\gamma^{-1} r(t) \mathbb{B} \subset R(t)$
ii) $F(t, x) \cap(\dot{\bar{x}}(t)+r(t) \mathbb{B}) \neq \emptyset$

for all $x \in \bar{x}(t)+\epsilon \overline{\mathbb{B}}$, a.e. $t \in[S, T]$.

Theorem 2.1 (Stratified necessary conditions). Let $\bar{x}($.$) be a W^{1,1}$ local minimizer for $(P)$ w.r.t. the radius multifunction $R($.$) . Assume (H1)-(H5). Then, there exist$ an arc $p(.) \in W^{1,1}\left([S, T] ; \mathbb{R}^{n}\right)$, a nonnegative number $\lambda$, a monotone non-decreasing function $\mu(.) \in N B V^{+}[S, T]$ and a $\mu$-integrable function $m($.$) , such that$

(i) $\lambda+\|p\|_{L^{\infty}}+\|\mu\|_{T . V .}=1$,

(ii) $\dot{p}(t) \in \operatorname{co}\left\{\eta:\left(\eta, p(t)+\int_{[S, t)} m(s) \mu(d s)\right) \in N_{G r F(t, .)}(\bar{x}(t), \dot{\bar{x}}(t))\right\}$ a.e.,

(iii) $\left(p(S),-\left[p(T)+\int_{[S, T]} m(s) \mu(d s)\right]\right) \in \lambda \partial \ell(\bar{x}(S), \bar{x}(T))+N_{E}(\bar{x}(S), \bar{x}(T))$,

(iv) $\left(p(t)+\int_{[S, t)} m(s) \mu(d s)\right) \cdot \dot{\bar{x}}(t) \geq\left(p(t)+\int_{[S, t)} m(s) \mu(d s) \cdot v\right.$

for all $v \in F(t, \bar{x}(t)) \cap R(t)$ a.e. $t \in[S, T]$,

(v) $m(t) \in \partial_{x}^{>} h(t, \bar{x}(t)) \mu$-a.e. and $\operatorname{supp}\{\mu\} \subset\{t: h(t, \bar{x}(t))=0\}$.

Here $\partial_{x}^{>} h(t, x)$ is the set

$$
\begin{array}{r}
\partial_{x}^{>} h(t, x):=\left\{\xi \mid \text { there exist } x_{i} \rightarrow x, t_{i} \rightarrow t, \xi_{i} \rightarrow \xi, \text { s. t., for each } i,\right. \\
\left.\nabla_{x} h\left(t_{i}, x_{i}\right) \text { exists, } \xi_{i}=\nabla_{x} h\left(t_{i}, x_{i}\right) \text { and } h\left(t_{i}, x_{i}\right)>0\right\} .
\end{array}
$$


We recall that the pathwise state constraint has been formulated as a functional inequality constraint $h(t, x(t)) \leq 0$, in which $h(.,$.$) is upper semicontinuous and$ uniformly Lipschitz continuous in the second variable. The versatility of this formulation was first noted and employed by Clarke in [5]. It covers the intrinsic formulation $x \in A(t)$ employed elsewhere in the literature (see, e.g. [1]), in which $A($.$) is a closed valued lower semicontinuous multifunction since, in this case, we$ may take $h(t, x):=d_{A(t)}(x)$. The way in which the hybrid subgradient $\partial_{x}^{>} h(.,$.$) is$ defined, via limits of gradients at points $\left(t_{i}, x_{i}\right)$ at which $h\left(t_{i}, x_{i}\right)>0$, is crucial to ensuring non-triviality of the necessary conditions in this case (it implies that $m($. satisfies $|m(t)|=1 \mu$-a.e.). Notice that the condition $\operatorname{supp}\{\mu\} \subset\{t: h(t, \bar{x}(t))=0\}$ is inserted for emphasis; it is actually implied by $m(t) \in \partial_{x}^{>} h(t, \bar{x}(t)) \mu$-a.e., since $\partial_{x}^{>} h(t, \bar{x}(t))$ is an empty set at those times $t$ where $h(t, \bar{x}(t))<0$. (See $([12]$, Chap. 9) for further discussion of this state constraint description.)

The first corollary we state is a variant on the stratified necessary conditions in which a 'bounded slope' condition, and an accompanying compatibility condition, replace (H4) and (H5). This set of necessary conditions, proved by Clarke [4] in the state constraint-free case, has been particularly useful as a starting point for deriving necessary conditions covering optimal control problems falling outside the formulation $(P)$, for example problems involving mixed state/velocity constraints $[7]$.

Corollary 2.2. The assertions of Theorem 2.1 remain valid when hypotheses $(H 4)$ and $(\mathrm{H} 5)$ are replaced by $\left(\mathrm{H}_{4}\right)^{*},(\mathrm{H} 5)^{*}$ :

$\left(H_{4}\right)^{*}$ There exist $\epsilon>0$ and $k(.) \in L^{1}(S, T)$ such that

$$
|x-\bar{x}(t)|<\epsilon, v \in F(t, x) \cap R(t),(\alpha, \beta) \in N_{G r F(t, .)}^{P}(x, v) \Rightarrow|\alpha| \leq k(t)|\beta| .
$$

$(H 5)^{*}$ For some $\omega>0$,

$$
\dot{\bar{x}}(t)+\omega k(t) \overline{\mathbb{B}} \subset R(t) .
$$

The second corollary provides necessary conditions under hypotheses which capture, as a special case, the 'pseudo-Lipschitz continuity condition with linear growth' hypothesis (1.4) as a special case. Here we show that the rate of growth can be traded against the integrability conditions on $k($.$) and its scaled higher powers.$ This corollary was proved earlier by Clarke [3] in the state constraint-free case.

Corollary 2.3. The assertions of Theorem 2.1 remain valid when $R(t)=\mathbb{R}^{n}$ a.e. and when hypotheses (H4) and (H5) are replaced by the hypothesis:

$\left(H_{4}\right)^{* *}$ There exist a number $\alpha>0$ and non-negative measurable functions $k($.$) and$ $\beta($.$) such that$

$$
k(.) \text { and } t \rightarrow \beta(t) k^{\alpha}(t) \text { are integrable }
$$

and, for each $N \geq 0$,

$$
F(t, x) \cap(\dot{\bar{x}}(t)+N \overline{\mathbb{B}}) \subset F\left(t, x^{\prime}\right)+\left(k(t)+\beta(t) N^{\alpha}\right)\left|x-x^{\prime}\right| \overline{\mathbb{B}}
$$

for all $x, x^{\prime} \in \bar{x}(t)+\epsilon \overline{\mathbb{B}}$ a.e. 


\section{Counter Examples and Discussion}

Suppose that the feasible $F$-trajectory $\bar{x}($.$) is a weak minimizer for problem (\mathrm{P})$ in the sense of the discussion of the introduction, i.e. there exists $r>0$ such that $\bar{x}($. is a minimizer w.r.t. admissible $F$-trajectories that satisfy

$$
|x(t)-\bar{x}(t)|<r \quad \text { and } \quad|\dot{x}(t)-\dot{\bar{x}}(t)|<r \quad \text { a.e.. }
$$

Suppose that hypotheses (H1)-(H3) are satisfied and also the stronger (global) form (1.1) of the Lipschitz continuity assumption (H4) is satisfied, but not (H5). Are the generalized Euler Lagrange condition, transversality condition and some restricted form of the Weierstrass condition still valid? This question can be addressed within the framework of stratified necessary conditions, by regarding $\bar{x}($.$) as a W^{1,1}$ local minimizer w.r.t. the constant radius multifunction $R(t)=\dot{\bar{x}}(t)+r \mathbb{B}$. Clarke ([3], pp. 46-47) showed through a counter-example that, if the 'tempered growth' condition (H5) is dropped, this is not the case. We provide another example illustrating the critical role of the tempered growth condition: if (H5) is dropped from the hypotheses of Thm. 2.1 then we cannot guarantee the simultaneous satisfaction of the generalized Euler Lagrange and the transversality conditions.

Example 1. Consider

$$
\left\{\begin{array}{l}
\text { Minimize }-x_{2}(1) \\
\text { over arcs } x \in W^{1,1}\left([0,1] ; \mathbb{R}^{2}\right) \quad \text { satisfying } \\
\dot{x}(t) \in F(t, x(t)) \text { a.e. } \\
x_{2}(0)=0
\end{array}\right.
$$

in which

$$
F(t, x):=\left\{\left(e_{1}, e_{2}\right) \in \mathbb{R}^{2} \mid e_{1}=0, e_{2}=k(t) x_{1}\right\} .
$$

Here, $k($.$) is any positive function in L^{1}(0,1)$ which is not essentially bounded.

Take an arbitrary number $r>0$. Then the $F$-trajectory $\left.\bar{x}()=.\left(\bar{x}_{1}=0, \bar{x}_{2}(.) \equiv 0\right)\right)$ is a minimizer w.r.t. the radius multifunction $R(.) \equiv r \mathbb{B}$. To see this, suppose to the contrary that there exists a feasible $F$-trajectory $x()=.\left(x_{1}(),. x_{2}().\right)$ with lower cost and such that

$$
\dot{x}(t) \in R(t) \quad \text { a.e.. }
$$

We have $x_{1}>\bar{x}_{1}=0$, whence $\dot{x}(t)=\left(0, x_{1} k(t)\right)$ is not essentially bounded. This implies that, on a set of positive measure, $\dot{x}(t) \notin R(t)$, in contradiction of (3.1).

Hypotheses (H1)-(H4) of Thm. 2.1 (with $h(.) \equiv-1)$ are satisfied for the above radius multifunction, but not (H5). This is because, for any $\gamma \in(0,1)$ and $\left(x_{1}, x_{2}\right) \in \mathbb{R}^{2}$ such that $x_{1} \neq 0$, the relation

$$
F(t, x)\left(=\left\{0, x_{1} k(t)\right\}\right) \notin \dot{\bar{x}}(t)+\gamma^{-1} r \mathbb{B}
$$

is satisfied on a set of positive measure, in violation of the tempered growth condition. 
Let us now examine the generalized Euler Lagrange and transversality conditions. They assert the existence of an arc $p()=.\left(p_{1}(),. p_{2}().\right)$ and $\lambda \geq 0$, not both zero, satisfying:

$$
\begin{aligned}
\dot{p}_{2}(t) & =0 \\
\dot{p}_{1}(t)+p_{2}(t) k(t) & =0
\end{aligned}
$$

and

$$
\begin{aligned}
p_{1}(0)=0, & p_{1}(1)=0 \\
& p_{2}(1)=\lambda .
\end{aligned}
$$

If $\lambda=0$ then also $p \equiv 0$, a contradiction. If, on the other hand, $\lambda>0$ then (3.2) implies

$$
p_{1}(1)=-\lambda \int_{0}^{1} k(t) d t<0
$$

in contradition of (3.3). We conclude that the generalized Euler Lagrange and transversality conditions cannot both be satisfied in this example.

Recall that the radius multifunction $R($.$) in Thm. 2.1$ is required to take open-set values. The second example tells us that the assertions of the theorem are no longer valid in general, if the radius function is assumed, instead, to take closed-set values.

Example 2. Consider the problem

$$
\left\{\begin{array}{l}
\text { Minimize }-x(1) \\
\text { over arcs } x \in W^{1,1}\left([0,1] ; \mathbb{R}^{1}\right) \quad \text { satisfying } \\
\dot{x}(t) \in F(t, x) \text { a.e. } \\
\text { and such that } x(0)=0
\end{array}\right.
$$

in which

$$
F(t, x)=\{0\} \cup\{1+|x|\} .
$$

Consider two different choices of radius multifunctions

$$
R(t) \equiv \mathbb{B} \quad \text { and } \quad R(t) \equiv \overline{\mathbb{B}}
$$

the first of which takes values open sets and the second closed sets. Then the $F$ trajectory $\bar{x}(.) \equiv 0$ is a minimizer w.r.t. either choice of radius multifunction. To confirm this assertion suppose, contrary to the assertion, that there exists a feasible $F$-trajectory $x($.$) w.r.t. the radius multifunction R($.

$$
\dot{x}(t) \in R(t)
$$

(for either choice of $R($.$) ), which has lower cost. Then x(1)>\bar{x}(1)=0$.

Define $\bar{t}:=\min \left\{t^{\prime} \in[0,1] \mid x(t)>0\right.$ for all $\left.t \in\left[t^{\prime}, 1\right]\right\}$. Then, since $x($.$) is continuous$ and $x(0)=0$, we have that $\bar{t}<1, x(\bar{t})=0$ and $x(t)>0$ for all $t \in(\bar{t}, 1]$. We deduce 
that $0<x(1)=0+\int_{\bar{t}}^{1} \dot{x}(s) d s$. But then there is a set of points $t \in(\bar{t}, 1]$, having positive measure, on which $\dot{x}(t) \neq 0$ and $\dot{x}(t)=1+|x(t)| \notin R(t)$. This is not possible, since $x($.$) is assumed to be feasible w.r.t. either choice of radius multifunction$ $R($.$) . So \bar{x}($.$) is indeed a minimizer w.r.t. to either radius multifunction.$

There is a unique set of non zero multipliers $(p(),. \lambda)$ (modulo scaling) satisfying the generalized Euler Lagrange and transversality conditions, namely $p(.) \equiv 1, \lambda=1$. Notice however that the Weierstrass condition

$$
p(t) \cdot \dot{\bar{x}}(t)=\max \{p(t) \cdot e \mid e \in F(t, \bar{x}(t)) \cap R(t)\} \quad \text { a.e. }
$$

is satisfied for $R(.) \equiv \mathbb{B}$ but not for $R(.) \equiv \overline{\mathbb{B}}$.

\section{Proof of Thm. 2.1 and Corollaries}

The proof of Thm. 2.1 will be the end-result of applying known, 'unstratified', necessary conditions for problem $(P)$ to a sequence of optimal control problems and passage to the limit. The known necessary conditions referred to here are:

Proposition 4.1. The assertions of Thm. 2.1 are valid when $R(t)=\mathbb{R}^{n}$ for all $t$. In this case, (H4) is the Lipschitz continuity condition on $F(t,$.$) : there exists$ $k(.) \in L^{1}$ and $\epsilon>0$ such that

$$
F\left(t, x^{\prime}\right) \subset F(t, x)+k(t)\left|x-x^{\prime}\right| \overline{\mathbb{B}} \quad \text { a.e. }
$$

for all $x, x^{\prime} \in \bar{x}(t)+\epsilon \overline{\mathbb{B}}$. (H5) is superfluous (as it is implied by (H4)) for a suitably adjusted $\epsilon$, and the qualifier ' $v \in F(t, \bar{x}(t)) \cap R(t)$ ' in the Weierstrass condition (iv) is interpreted as ' $v \in F(t, \bar{x}(t))$ '.

Proof. See ([12], Thm 10.3.1). Notice that, if (4.1) is satisfied, then hypothesis $(G 2)$ of this theorem is automatically satisfied (for $\beta=0$ and the same $k($.$) and \epsilon$.)

We observe that a number of reductions can be performed at the outset to simplify the analysis (cf. [3]).

Lemma 4.2. The assertions of the Thm. 2.1 are valid in general if they can be verified in the special case when

$(a): \bar{x}(.) \equiv 0$,

(b): The interval $[S, T]=[0,1]$ and the function $r($.$) in hypothesis (H 5)$ is a positive constant $r(.) \equiv r_{0}$ for some constant $r_{0}>0$,

(c): $\bar{x}($.$) is a L^{\infty}$ local minimizer w.r.t. $R($.$) , and not merely a W^{1,1}$ local minimizer, i.e. $\bar{x}($.$) minimizes the cost over all feasible F$-trajectories $x($.$) satisfying$ $\|x(.)-\bar{x}(.)\|_{L^{\infty}} \leq \epsilon$, for some fixed $\epsilon$. 
The justification for these reductions is straightforward. We need to show, in each case, that the data can be transformed so that the relevant additional hypothesis is satisfied and that the assertions of the (special case of the) theorem, applied to the transformed problem, can be interpreted as an assertion of the desired properties of the original problem. (a) is dealt with by a translation of $\bar{x}($.$) to the origin,$ following which the multifunctions $F(t, x)$ and $R(t)$ become $F(t, \bar{x}(t)+x)-\{\dot{\bar{x}}(t)\}$ and $R(t)-\{\dot{\bar{x}}(t)\}$. (b) is dealt with by introducing a new independent variable $\tau$ related to the original time variable $t$ according to $\tau(t)=c \int_{S}^{t} r(s) d s$, in which $c$ is a positive constant adjusted so that $\tau(T)=1$. Concerning (c) we observe that, if $\bar{x}($. is merely a local $W^{1,1}$ minimizer, it is a $L^{\infty}$ minimizer for a modified problem into which is introduced the additional constraints:

$$
|x(0)-\bar{x}(0)| \leq \epsilon \text { and } \int_{0}^{1}|\dot{x}(t)-\dot{\bar{x}}(t)| d t \leq \epsilon .
$$

The problem with additional constraints can be treated as a problem of the form $(P)$ by means of state augmentation. In view of the fact that the extra conditions (4.2) are not active at $x()=.\bar{x}($.$) , the necessary conditions for this related problem$ imply the desired necessary conditions for the original problem. Henceforth then we assume (a)-(c).

We next define and list salient properties of a well-known construct from convex analysis, namely the gauge function $g($.$) of an open convex set \Gamma$ containing the origin. The role of the gauge function is to quantify the extent to which a point lies in $\Gamma$.

Lemma 4.3. Take an open convex set $\Gamma$ containing the open ball of radius $r_{0}$ centered at the origin. Define the function $g():. \mathbb{R}^{n} \rightarrow[0,+\infty)$ to be

$$
g(x):=\inf \left\{\lambda>0: \lambda^{-1} x \in \Gamma\right\} .
$$

Then $g($.$) has the following properties:$

1. $g(c x)=c g(x)$ for every constant $c>0$.

2. $g(x)=0$ if and only if $x=0$.

3. $g($.$) is Lipschitz with Lipschitz constant 1 / r_{0}$.

4. $\Gamma=\left\{x \in \mathbb{R}^{n}: g(x)<1\right\}$.

In view of $(H 5)$, we may choose $\eta \in(0,0.5)$ such that, for all $x \in \epsilon \overline{\mathbb{B}}$ and $N>\frac{r_{0}}{1-2 \eta}$,

$$
(1-2 \eta)^{-1} r_{0} \mathbb{B} \subset R_{N}(t) \text { and } F(t, x) \cap r_{0} \mathbb{B} \neq \emptyset, \text { a.e.. }
$$

Here

$$
R_{N}(t):=R(t) \cap N \mathbb{B} .
$$


Now take $g_{t}($.$) to be the gauge function of R_{N}(t)$.

Following Clarke $[3,4]$ we embed information about $F(.,$.$) in a new multi-function$ $\widetilde{F}(.,):.[0,1] \times \mathbb{R}^{n} \leadsto \mathbb{R}^{n} \times \mathbb{R}$ taking values subsets in $\mathbb{R}^{n+1}$ : For each $(t, x) \in$ $[0,1] \times \mathbb{R}^{n}$,

$$
\widetilde{F}(t, x):= \begin{cases}\left\{(\xi e, \xi) \mid \xi \in[0,1], e \in F(t, x), g_{t}(e) \leq 1-\xi \eta\right\} & \text { if } x \in \epsilon \overline{\mathbb{B}} \\ \{0\} & \text { otherwise. }\end{cases}
$$

Lemma 4.4. $\widetilde{F}(.,$.$) takes values non-empty sets. For a.e. t, \widetilde{F}(t,$.$) has closed$ graph. Furthermore,

$$
\widetilde{F}(t, x) \subset \widetilde{F}\left(t, x^{\prime}\right)+\widetilde{k}(t)\left|x-x^{\prime}\right| \overline{\mathbb{B}}, \text { for all } x, x^{\prime} \in \epsilon \overline{\mathbb{B}} \text {, a.e. },
$$

where $\widetilde{k}(t)=\left(1+\frac{N+1}{\eta r_{0}}\right) k(t)$.

Proof. The fact that $\widetilde{F}(.,$.$) takes values non-empty sets follows from (4.3). Showing$ that $\widetilde{F}(t,$.$) has closed graph is a simple exercise. To establish that \widetilde{F}(t,$.$) is Lipschitz$ continuous on $\epsilon \overline{\mathbb{B}}$ with the stated Lipschitz constant, take $x, x^{\prime} \in \epsilon \overline{\mathbb{B}}$ and $(\xi e, \xi) \in$ $\widetilde{F}(t, x)$. We have two possibilities:

A. $\xi \leq \frac{k(t)\left|x-x^{\prime}\right|}{\eta r_{0}}$ : By (H5) there exists a velocity $e^{\prime} \in F\left(t, x^{\prime}\right)$ such that $g_{t}\left(e^{\prime}\right)<1$.

Then $(0,0) \in \widetilde{F}\left(t, x^{\prime}\right)$ and

$$
|(\xi e, \xi)-(0,0)| \leq \frac{k(t)\left|x-x^{\prime}\right|}{\eta r_{0}}(|e|+1) \leq \widetilde{k}(t)\left|x-x^{\prime}\right| .
$$

B. $\xi>\frac{k(t)\left|x-x^{\prime}\right|}{\eta r_{0}}$ : By $(\mathrm{H} 4)$ there exists a velocity $e^{\prime} \in F\left(t, x^{\prime}\right)$ such that $\mid e-$ $e^{\prime}|\leq k(t)| x-x^{\prime} \mid$. Let $\xi^{\prime}=\xi-\frac{k(t)\left|x-x^{\prime}\right|}{\eta r_{0}}$. Then $\xi^{\prime} \in(0,1]$. Noting that $r_{0}^{-1} k(t)\left|x-x^{\prime}\right|=\eta\left(\xi-\xi^{\prime}\right)$ we see that

$$
g_{t}\left(e^{\prime}\right) \leq g_{t}(e)+\frac{1}{r_{0}}\left|e-e^{\prime}\right| \leq(1-\xi \eta)+\frac{1}{r_{0}} k(t)\left|x-x^{\prime}\right|=\left(1-\xi^{\prime} \eta\right) .
$$

It follows that $\left(\xi^{\prime} e^{\prime}, \xi^{\prime}\right) \in \widetilde{F}\left(t, x^{\prime}\right)$. It remains to observe that

$$
\left|(\xi e, \xi)-\left(\xi^{\prime} e^{\prime}, \xi^{\prime}\right)\right| \leq\left|\xi e-\xi^{\prime} e^{\prime}\right|+\left|\xi-\xi^{\prime}\right| \leq\left(\xi-\xi^{\prime}\right)(|e|+1)+\left|e-e^{\prime}\right| \leq \widetilde{k}(t)\left|x-x^{\prime}\right| .
$$

For each $t \in[0,1]$ define now the function $\phi_{t}: \mathbb{R}^{n} \rightarrow \mathbb{R}_{+}$to be

$$
\phi_{t}(e):=\max \left\{\frac{r_{0}}{2 N}\left(g_{t}(e)-(1-2 \eta)\right), 0\right\} .
$$

The following properties of $\phi_{t}($.$) will be of particular significance$

$$
\begin{aligned}
& \phi_{t}(e)=0 \quad \text { iff } \quad g_{t}(e) \leq 1-2 \eta \\
& \left|\phi_{t}(e)-\phi_{t}\left(e^{\prime}\right)\right| \leq \frac{1}{2 N}\left|e-e^{\prime}\right| \\
& \phi_{t}(e) \geq \frac{\eta r_{0}}{4 N} \quad \text { if } \quad g_{t}(e)>1-\frac{3}{2} \eta .
\end{aligned}
$$


With these definitions and constructions behind us, we are ready to start the proof of Thm. 2.1. This will hinge on properties of a sequence of optimization problems $\left(P_{i}\right)$, involving the arbitrary sequence $\epsilon_{i} \downarrow 0$. Define

$$
J_{i}(x(.), y(.)):=\max \left\{\ell(x(0), x(1))-\ell(0,0)+\epsilon_{i}^{2}, 1-y(1)\right\}+\int_{0}^{1} \phi_{t}(\dot{x}(t)) d t
$$

and consider the optimal control problem

$$
\left(P_{i}\right) \quad\left\{\begin{array}{l}
\text { Minimize } J_{i}(x(.), y(.)) \\
\text { over }(x(.), y(.)) \in W^{1,1}\left([0,1] ; \mathbb{R}^{n} \times \mathbb{R}\right) \text { satisfying } \\
(\dot{x}(t), \dot{y}(t)) \in \widetilde{F}(t, x(t)) \quad \text { for a.e. } t \in[0,1] \\
(x(0), x(1)) \in E, \quad y(0)=0 \\
h(t, x(t)) \leq 0 \text { for all } t \in[0,1]
\end{array}\right.
$$

Denote by $\mathcal{S}$ the set of feasible $\widetilde{F}$-trajectories for $\left(P_{i}\right)$. Then $J_{i}(x(),. y()$.$) defines a$ continuous function on $\mathcal{S}$ with respect to the $W^{1,1}$ metric. Noting that $J_{i}$ is nonnegative, we see that $(\bar{x}(.) \equiv 0, \bar{y}(t) \equiv t)$ is an $\epsilon_{i}^{2}$ solution of $\left(P_{i}\right)$. It follows then from Ekeland's Theorem that there exists $\left(x_{i}(),. y_{i}().\right) \in \mathcal{S}$ such that

$$
\left\|x_{i}\right\|_{W^{1,1}\left([0,1] ; \mathbb{R}^{n}\right)} \leq \epsilon_{i} \quad \text { and } \quad \int_{0}^{1}\left|\dot{y}_{i}(t)-1\right| d t \leq \epsilon_{i}
$$

and $\left(x_{i}(),. y_{i}().\right)$ is a minimizer for the perturbed problem:

$$
\left(\widetilde{P}_{i}\right)\left\{\begin{array}{c}
\text { Minimize } \widetilde{J}_{i}\left(x_{i}(.), y_{i}(.)\right):=J_{i}\left(x_{i}(.), y_{i}(.)\right) \\
\quad+\epsilon_{i}\left|x(0)-x_{i}(0)\right|+\epsilon_{i} \int_{0}^{1}\left(\left|\dot{x}(t)-\dot{x}_{i}(t)\right|+\left|\dot{y}(t)-\dot{y}_{i}(t)\right|\right) d t \\
\text { over }(x(.), y(.)) \in \mathcal{S} .
\end{array}\right.
$$

Using the usual state augmentation techniques to eliminate the integral terms in the cost, we can reformulate $\left(\widetilde{P}_{i}\right)$ as an optimization problem to which the already known special case Prop. 4.1 of the theorem is applicable. Note, in particular, that the multifunction involved $\widetilde{F}(t,$.$) is Lipschitz continuous. Bearing in mind that the$ multifunction $\widetilde{F}(t,$.$) does not depend on y$, and $y$ does not appear in the integral cost term, we observe that the costate $q$ corresponding to the $y$-variable is in fact constant. Prop. 4.1 tells us then that there exist, for each $i, p_{i}(.) \in W^{1,1}\left([0,1] ; \mathbb{R}^{n}\right)$, $q_{i} \in \mathbb{R}, \lambda_{i} \geq 0, \mu_{i} \in N B V^{+}[0,1], \chi_{i} \in[0,1]$ and a Borel measurable function $m_{i}:[0,1] \rightarrow \mathbb{R}^{n}$ such that

(A1) $q_{i}+\left\|p_{i}\right\|_{L^{\infty}}+\left\|\mu_{i}\right\|_{T . V .}+\lambda_{i}=1$.

$$
\begin{aligned}
\dot{p}_{i}(t) \in \operatorname{co}\left\{\nu:\left(\nu, p_{i}(t)+\int_{[0, t)}\right.\right. & \left.m_{i}(s) \mu_{i}(d s), q_{i}\right) \in N_{G r \widetilde{F}(t, .)}\left(x_{i}(t), \dot{x}_{i}(t), \dot{y}_{i}(t)\right) \\
& \left.+\{0\} \times \lambda_{i} \epsilon_{i} \mathbb{B} \times \lambda_{i} \epsilon_{i} \mathbb{B}+\{0\} \times \lambda_{i} \partial \phi_{t}\left(\dot{x}_{i}(t)\right) \times\{0\}\right\} .
\end{aligned}
$$

$$
\begin{aligned}
\left(p_{i}(0),-\left[p_{i}(1)+\int_{[0,1]} m_{i}(s) \mu_{i}(d s)\right]\right) & \in N_{E}\left(x_{i}(0), x_{i}(1)\right) \\
& +\lambda_{i} \epsilon_{i} \mathbb{B} \times\{0\}+\lambda_{i} \chi_{i} \partial \ell\left(x_{i}(0), x_{i}(1)\right) .
\end{aligned}
$$

(A4) $q_{i}=\lambda_{i}\left(1-\chi_{i}\right)$. Observe that in the case when $\ell\left(x_{i}(0), x_{i}(1)\right)-\ell(0,0)+\epsilon_{i}^{2}>$ $1-y_{i}(1)$ we have that $\chi_{i}=1$. 
(A5)

$$
\begin{aligned}
& \text { A5) }\left(p_{i}(t)+\int_{[0, t)} m_{i}(s) \mu_{i}(d s)\right) \cdot \dot{x}_{i}(t)+q_{i} \dot{y}_{i}(t)-\lambda_{i} \phi_{t}\left(\dot{x}_{i}(t)\right) \geq \\
& \left(p_{i}(t)+\int_{[0, t)} m_{i}(s) \mu_{i}(d s)\right) \cdot(\xi e)+q_{i} \xi-\lambda_{i} \phi_{t}(\xi e)-\lambda_{i} \epsilon_{i}\left(\left|\xi e-\dot{x}_{i}(t)\right|+\left|\xi-\dot{y}_{i}(t)\right|\right)
\end{aligned}
$$

for all $(\xi e, \xi) \in \widetilde{F}\left(t, x_{i}(t)\right)$.

(A6) $m_{i}(t) \in \partial_{x}^{>} h\left(t, x_{i}(t)\right) \mu_{i}$-a.e. and $\operatorname{supp}\left\{\mu_{i}\right\} \subset\left\{t: h\left(t, x_{i}(t)\right)=0\right\}$.

By (4.6) we can arrange by subsequence extraction that

$$
\dot{x}_{i}(t) \rightarrow 0 \quad \text { a.e., } \quad \dot{y}_{i}(t) \rightarrow 1 \text { a.e.. }
$$

It may be deduced from (A1) and (A2) and the Lipschitz continuity properties of $\widetilde{F}(t,$.$) that the p_{i}($.$) 's are uniformly bounded and the \dot{p}_{i}($.$) 's are majorized by$ a common integrable function. We may conclude that, following an extraction of subsequences, $p_{i} \rightarrow p$ uniformly in $W^{1,1}$ and $\dot{p}_{i} \rightarrow \dot{p}$ weakly in $L^{1}, q_{i} \rightarrow q, \lambda_{i} \rightarrow \lambda$, $\mu_{i} \rightarrow \mu$ weakly*,$m_{i} d \mu_{i} \rightarrow m d \mu$ weakly* and $\chi_{i} \rightarrow \chi$ for multipliers $p(), q,. \lambda, \mu, \chi$ such that

$$
q+\|p\|_{L^{\infty}}+\|\mu\|_{T . V .}+\lambda=1 .
$$

We have derived a set of conditions satisfied by $x_{i}(),. p_{i}(),. q_{i}, \mu_{i}$ and $\lambda_{i}$, for each $i$. The convergence analysis of ([12], Thm. 2.5.3 and Prop. 2.6.1) permits us to deduce the following properties of their limits:

$$
\begin{aligned}
& \dot{p}(t) \in \operatorname{co}\left\{\nu:\left(\nu, p(t)+\int_{[0, t)} m(s) \mu(d s), q\right) \in N_{G r \widetilde{F}(t, .)}(0,0,1)\right\} \text { a.e.. } \\
& \left(p(0),-\left[p(1)+\int_{[0,1]} m(s) \mu(d s)\right]\right) \in N_{E}(0,0)+\lambda \chi \partial \ell(0,0) . \\
& q \geq\left(p(t)+\int_{[0, t)} m(s) \mu(d s)\right) \cdot(\xi e)+q \xi-\lambda \phi_{t}(\xi e) \text { a.e. } \\
& \text { for all }(\xi e, \xi) \in \widetilde{F}(t, 0)
\end{aligned}
$$

(B4) $m(t) \in \partial_{x}^{>} h(t, 0) \mu$-a.e. and $\operatorname{supp}\{\mu\} \subset\{t: h(t, 0)=0\}$.

Note next the following lemma relating the normal cones to the graphs of $\widetilde{F}(t,$.$) and$ $F(t,$.$) . The straightforward proof, that depends on establishing the relationship for$ proximal normals and then exploiting the interpretation of normal vectors as limits of proximal normals at neighbouring points, is omitted.

Lemma 4.5. Take any $x \in \epsilon \mathbb{B}$. If a vector $(\alpha, \beta, \gamma) \in N_{G r \widetilde{F}(t, .)}(x, \xi f, \xi)$, where $g_{t}(f) \leq 1-2 \eta$, then $(\alpha, \xi \beta) \in N_{G r F(t, .)}(x, f)$.

It follows from (B1) that

$(\mathrm{C} 1) \dot{p}(t) \in \operatorname{co}\left\{\nu:\left(\nu, p(t)+\int_{[0, t)} m(s) \mu(d s)\right) \in N_{G r F(t, .)}(0,0)\right\}$ a.e..

Recall that $\phi_{t}(e)=0$ if $g_{t}(e) \leq 1-2 \eta$ and, in particular, that $\phi_{t}(0)=0$. Also $(1-2 \eta) R_{N}(t)=\left\{e \mid g_{t}(e)<1-2 \eta\right\}$. It follows from (B3), upon setting $\xi=1$, that 


$$
\left(p(t)+\int_{[0, t)} m(s) \mu(d s)\right) \cdot e \leq 0
$$

for all $e \in F(t, 0) \cap((1-2 \eta)(R(t) \cap N \mathbb{B}))$.

Define $\widetilde{\lambda}=\lambda \chi$. Then we see from (C1), (B2), (C2), (B4) and (4.7) that a restricted form of the assertions of Theorem 2.1, in which the Weierstrass condition holds for $v$ 's in the subset $F(t, 0) \cap((1-2 \eta)(R(t) \cap N \mathbb{B}))$ of $F(t, 0) \cap R(t)$, are satisfied, provided we can show that

$$
\|p\|_{L^{\infty}}+\|\mu\|_{T . V .}+\widetilde{\lambda} \neq 0
$$

To confirm this 'non-triviality' relation, we make essential use of the Weierstrass condition (A5) for each $i$. Let $\mathcal{I} \in[0,1]$ be the set of full measure comprising $t \in[0,1]$ such that $\left(\dot{x}_{i}(t), \dot{y}_{i}(t)\right) \in \widetilde{F}\left(t, x_{i}(t)\right)$, the Weierstrass condition (A5) and hypotheses (H4) and (H5) are valid for each $i$. We can always arrange by extracting a suitable subsequence that one of the following three cases applies:

Case 1: $\dot{y}_{i}(t)=1$ a.e., in which case $y_{i}(1)=1$, for all $i$.

Case 2: There exist $\left\{t_{i}\right\} \subset \mathcal{I}$ and $\alpha \in[0,1]$ such that $t_{i} \uparrow 1$ and $\dot{y}_{i}\left(t_{i}\right)<1-\alpha$ for all $i$.

Case 3: There exists $\left\{t_{i}\right\} \subset \mathcal{I}$ such that $t_{i} \uparrow 1, \dot{y}_{i}\left(t_{i}\right)<1$ for each $i$, and $\dot{y}_{i}\left(t_{i}\right) \rightarrow 1$.

Case 1: if $y_{i}(t) \equiv 1$ for all $i$ then, for each $i, x_{i}($.$) is actually an F-trajectory. Since$ it satisfies the constraints of problem $(\mathrm{P})$ we must have $\ell\left(x_{i}(0), x_{i}(1)\right)-\ell(0,0)+\epsilon_{i}^{2}>$ $\ell\left(x_{i}(0), x_{i}(1)\right)-\ell(0,0) \geq 0=1-y_{i}(1)$. It follows that the term $\left(1-y_{i}(1)\right)$ is not active in the evaluation of 'max' operation in the cost. Consequently $\chi_{i}=1$ and $q_{i}=0$. So $(q, \lambda)=(0, \widetilde{\lambda})$ and it follows from (4.7) that

$$
\|p\|_{L^{\infty}}+\|\mu\|_{T . V .}+\widetilde{\lambda}=1 .
$$

Case 2: Consider the Weierstrass condition (A5). According to (H5) since (1 $2 \eta)^{-1} r_{0} \mathbb{B} \subset R_{N}(t)$ and $F\left(t, x_{i}(t)\right) \cap r_{0} \mathbb{B} \neq \emptyset$ a.e., we can choose $e \in F\left(t, x_{i}(t)\right)$ such that $g_{t}(e)<1-2 \eta$. Set $\xi=1$. We have for each $i$

$$
\begin{aligned}
\left(p_{i}\left(t_{i}\right)+\right. & \left.\int_{\left[0, t_{i}\right)} m_{i}(s) \mu_{i}(d s)\right) \cdot \dot{x}_{i}\left(t_{i}\right)+q_{i} \dot{y}_{i}\left(t_{i}\right)-\lambda_{i} \phi_{t_{i}}\left(\dot{x}_{i}\left(t_{i}\right)\right) \\
& \geq\left(p_{i}\left(t_{i}\right)+\int_{\left[0, t_{i}\right)} m_{i}(s) \mu_{i}(d s)\right) \cdot e+q_{i}-\lambda_{i} \epsilon_{i}\left(\left|e-\dot{x}_{i}\left(t_{i}\right)\right|+\left|1-\dot{y}_{i}\left(t_{i}\right)\right|\right) .
\end{aligned}
$$

We obtain in the limit as $i \rightarrow+\infty$ the relation

$$
\left(\|p\|_{L^{\infty}}+k_{h}\|\mu\|_{T . V .}\right) N>q \alpha .
$$

If $q \neq 0$ then $\|p\|_{L^{\infty}}+k_{h}\|\mu\|_{T . V .}>0$. If on the other hand $q=0$, then

$$
(q, \lambda)=(0, \tilde{\lambda})
$$

and by (4.7),

$$
\|p\|_{L^{\infty}}+\|\mu\|_{T . V .}+\widetilde{\lambda}=1 .
$$


Note that, in this case, $\lambda=0$ implies $\|p\|_{L^{\infty}}+\|\mu\|_{T . V .}=1$.

Case 3: By definition of $\widetilde{F}(.,$.$) we know that \dot{x}_{i}(t)=\dot{y}_{i}(t) e_{i}$ for some $e_{i} \in F\left(t, x_{i}(t)\right)$ such that $g_{t}\left(e_{i}\right) \leq 1-\dot{y}_{i}(t) \eta$. By extracting subsequences we can arrange that either of the following situations must arise:

$$
\begin{aligned}
& g_{t_{i}}\left(e_{i}\right)<1-\dot{y}_{i}\left(t_{i}\right) \eta \\
& g_{t_{i}}\left(e_{i}\right)=1-\dot{y}_{i}\left(t_{i}\right) \eta
\end{aligned}
$$

for each $i$. Suppose first (4.8) is true. Take any $\xi \in\left(\dot{y}_{i}\left(t_{i}\right), 1\right]$ such that $g_{t_{i}}\left(e_{i}\right) \leq 1-\eta \xi$ and fix $e=e_{i}$. Then, from (A5) again,

$$
\begin{aligned}
\left(p_{i}\left(t_{i}\right)+\int_{\left[0, t_{i}\right)} m_{i}(s) \mu_{i}(d s)\right) \cdot\left(e_{i}\left(\dot{y}_{i}\left(t_{i}\right)-\xi\right)\right)+ & q_{i}\left(\dot{y}_{i}\left(t_{i}\right)-\xi\right) \geq \lambda_{i}\left(\phi_{t_{i}}\left(\dot{y}_{i}\left(t_{i}\right) e_{i}\right)-\phi_{t_{i}}\left(\xi e_{i}\right)\right) \\
& -\lambda_{i} \epsilon_{i}\left(\left|\xi e_{i}-\dot{y}_{i}\left(t_{i}\right) e_{i}\right|+\left|\xi-\dot{y}_{i}\left(t_{i}\right)\right|\right) .
\end{aligned}
$$

Since $\left|e_{i}\right| \leq N$ and $\phi_{t}($.$) has Lipschitz constant 1 / 2 N$,

$$
\left|\phi_{t_{i}}\left(\dot{y}_{i}\left(t_{i}\right) e_{i}\right)-\phi_{t_{i}}\left(\xi e_{i}\right)\right| \leq \frac{1}{2}\left|\xi-\dot{y}_{i}\left(t_{i}\right)\right| .
$$

Estimating terms as before, dividing across the inequality by $\left(\xi-\dot{y}_{i}\left(t_{i}\right)\right)$ and passage to the limit gives

$$
\left(\|p\|_{L^{\infty}}+k_{h}\|\mu\|_{T . V .}\right) N+\frac{1}{2} \lambda \geq q .
$$

But $\lambda=\chi \lambda+(1-\chi) \lambda=\widetilde{\lambda}+q$, so

$$
\left(\|p\|_{L^{\infty}}+k_{h}\|\mu\|_{T . V .}\right) N+\frac{1}{2} \widetilde{\lambda} \geq \frac{1}{2} q .
$$

It follows that, if $q>0,\left(\|p\|_{L^{\infty}}+k_{h}\|\mu\|_{T . V .}\right) N+\frac{1}{2} \widetilde{\lambda}>0$. If, on the other hand, $q=0$, then $\lambda=\widetilde{\lambda}$ and $\|p\|_{L^{\infty}}+\|\mu\|_{T . V .}+\widetilde{\lambda}=1$, by $(4.7)$.

Suppose finally that (4.9) is true. Take $\xi=1$ and take $e$ to be a point $e_{0} \in$ $F\left(t_{i}, x_{i}\left(t_{i}\right)\right) \cap N \mathbb{B}$ such that $g_{t_{i}}\left(e_{0}\right) \leq 1-2 \eta$. Then, still from (A5), we have

$$
\left(\left\|p_{i}\right\|_{L^{\infty}}+k_{h}\left\|\mu_{i}\right\|_{T . V .}\right) 2 N+\lambda_{i} \epsilon_{i} 3 N \geq q_{i}\left(1-\dot{y}\left(t_{i}\right)\right)+\lambda_{i} \phi_{t_{i}}\left(\dot{x}_{i}\left(t_{i}\right)\right) .
$$

Since $g_{t_{i}}\left(\dot{x}_{i}\left(t_{i}\right)\right)=\dot{y}_{i}\left(t_{i}\right) g_{t_{i}}\left(e_{i}\right)=\dot{y}_{i}\left(t_{i}\right)\left(1-\dot{y}_{i}\left(t_{i}\right) \eta\right) \rightarrow 1-\eta$ (recall that in case 3 , $\dot{y}_{i}\left(t_{i}\right) \rightarrow 1$ ), by (4.4) and (4.9) we have that $\phi_{t_{i}}\left(\dot{x}_{i}\left(t_{i}\right)\right) \geq \frac{\eta r_{0}}{4 N}$ for all $i$ big enough. Thus

$$
\left(\|p\|_{L^{\infty}}+k_{h}\|\mu\|_{T . V .}\right) 2 N \geq \lambda \frac{\eta r_{0}}{4 N}=(\widetilde{\lambda}+q) \frac{\eta r_{0}}{4 N} .
$$

If $q>0$ then $\|p\|_{L^{\infty}}+k_{h}\|\mu\|_{T . V .}>0$. If $q=0$, then $(q, \lambda)=(0, \tilde{\lambda})$ and $\|p\|_{L^{\infty}}+$ $\|\mu\|_{T . V .}+\widetilde{\lambda}=1$ follows by (4.7).

The foregoing demonstrates that, in all cases the multipliers arising in the limit are non-trivial. We may scale them so that

$$
\|p\|_{L^{\infty}}+\|\mu\|_{T . V .}+\widetilde{\lambda}=1 .
$$


Reviewing (C1), (B2), (C2), (B4) and (4.10), we see that all assertions of the theorem has been proved except that the Weierstrass condition is satisfied, a.e., only with respect to $e$ 's satisfying

$$
e \in F(t, 0) \cap((1-2 \eta)(R(t) \cap N \mathbb{B})) .
$$

Now take $\eta_{i} \downarrow 0$ and $N_{i} \rightarrow \infty$. (B1), (B2), (B4), (C1), (C2) and (4.10) are satisfied with $(p, \mu, \widetilde{\lambda}, m)$ replaced by some $\left(p_{i}, \mu_{i}, \widetilde{\lambda}_{i}, m_{i}\right)$. It is important to note that the Euler Lagrange inclusion (B1), whose right side is evaluated at $\bar{x} \equiv 0$, ensures that the integral bound on the $\dot{p}_{i}($.$) 's is independent of i$. So we may extract subsequences that are in relevant respects convergent and yield in the limit multipliers $(p, \mu, \widetilde{\lambda}, m)$ with the required properties. The Weierstrass condition is now strengthened to allow

$$
e \in F(t, \bar{x}(t)) \cap R(t) .
$$

The fact that, for each $t \in[0,1], R(t)$ is an open set is required in this analysis.

\section{Proof of Cor. 2.2}

Proof. We assume that $\bar{x}(.) \equiv 0$. As earlier remarked, there is no loss of generality in so doing. Clarke [4, Prop. 2] shows that if a multifunction $F(.,$.$) satisfies the$ bounded slope condition $(\mathrm{H} 4)^{*}$ and condition $(\mathrm{H} 5)^{*}$ then, for any $\eta \in(0,1)$, it satisfies also the pseudo Lipschitz condition (H4) w.r.t. the radius multi-function $(1-\eta) R($.$) and with the same Lipschitz constant k($.$) , following, possibly, a reduction$ in the size $\epsilon$. Also, (H5) in this setting is a consequence of (H4) and (H5)* if, as we can always arrange, $k(t) \geq 1$ a.e and $\epsilon \leq \omega$. Application of Thm. 2.1 now yields the assertions of Cor. 2.2, except that the Weierstrass condition applies only w.r.t. the restricted radius multi-function $(1-\eta) R($.$) . The full set of necessary conditions is$ provided by taking any sequence $\eta_{i} \downarrow 0$, obtaining the necessary conditions for each $i$ with reference to $\left(1-\eta_{i}\right) R($.$) , and passage to the limit.$ 


\section{Proof of Cor. 2.3}

Proof. We may assume that $\epsilon$ has been chosen such that $\epsilon<1$ and $k(t) \geq k_{0}$ for some $k_{0}>0$. Setting $N=0$ in $(\mathrm{H} 4)^{* *}$ yields

$$
\dot{\bar{x}}(t) \in F(t, x)+2 \epsilon k(t) \overline{\mathbb{B}} \quad \text { for all } x \in \bar{x}(t)+\epsilon \overline{\mathbb{B}} \text { a.e.. }
$$

Take any $m \geq 4$. Setting $N=m k(t)$ also yields

$$
F(t, x) \cap(\dot{\bar{x}}(t)+m k(t) \mathbb{B}) \subset F\left(t, x^{\prime}\right)+\left(k(t)+m^{\alpha} \beta(t) k^{\alpha}(t)\right) \overline{\mathbb{B}},
$$

for all $x, x^{\prime} \in \bar{x}(t)+\epsilon \overline{\mathbb{B}}$ a.e..

We see that (H4) and (H5) are satisfied for $\gamma=\frac{1}{2}, r(t)=2 k(t)$, the radius function $R(t)=\dot{\bar{x}}(t)+m k(t) \mathbb{B}$ and Lipschitz constant $\left(k(t)+m^{\alpha} \beta(t) k^{\alpha}(t)\right) \in L^{1}$. Apply Thm. 2.1 to obtain the assertions of the corollary, but in which the Weierstrass condition is valid only for $e \in F(t, \bar{x}(t)) \cap R(t)$. Now take $m_{i} \uparrow \infty$. Replacing $m$ by $m_{i}$ for each $i$, we obtain the full assertions of the corollary from these conditions, in the limit as $i \rightarrow \infty$, as in our earlier analysis.

\section{Acknowledgment}

This work was supported in part by the European Union, under the 7th Framework Programme FP7-PEOPLE- 2010-ITN Grant agreement number 264735-SADCO, and in part by EPSRC grant EP/G066477/1.

\section{References}

[1] A. V. Arutyunov and S. M. Aseev. Investigaiton of the degeneracy phenomenon of the maximum principle for optimal control problems with state constraints. SIAM J. Control and Optim., 35, 1977, pp. 930-952.

[2] P. Bettiol, A. Boccia and R. B. Vinter, Stratified necessary conditions for unbounded differential inclusions with state constraints, Proc. CDC 2012, Hawaii, Dec. 2012, pp. 7703-7707.

[3] F. H. Clarke. Necessary Conditions in Dynamic Optimization. Memoirs of the American Mathematical Society No. 173, 2005.

[4] F. H. Clarke. A General Theorem on Necessary Conditions In Optimal Control. Discrete and Continuous Dynamical Systems vol.29, No.2, February 2011, pp. 485-503.

[5] F. H. Clarke. Optimization and Nonsmooth Analysis. Wiley-Interscience, New York, 1983. Republished as vol. 5 of "Classics in Applied Mathematic," SIAM, 1990.

[6] F. H. Clarke, Y. S. Ledyaev, R. J. Stern and P. R. Wolenski. Nonsmooth Analysis and Control Theory. Graduate texts in Mathematics vol. 178, Springer Verlag, New York, 1998. 
[7] F. H. Clarke and M. do R. De Pinho. Optimal Control Problems with Mixed Constraints. SIAM J. Control Optim. 48, 2010, pp. 4500-4524.

[8] F. H. Clarke, Y. S. Ledyaev, and M. d. R. de Pinho, An extension of the Schwarkkopf multiplier rule in optimal control, SIAM J. Control and Optim. 49, 2011, pp. 599-610.

[9] A. Ioffe. Euler-Lagrange and Hamiltonian Formalisms in Dynamic Optimization. Trans. Amer. Math. Soc. 349, 1997, pp.2871-2900.

[10] P. Loewen and R.T. Rockafellar. Optimal control of unbounded differential inclusions. SIAM J. Control Optimization 32, 1994, pp. 442-470.

[11] R. T. Rockafellar and R. Wets. Variational Analysis. Springer-Verlag, New York, 1998.

[12] R. B. Vinter. Optimal Control. Birkhäuser, Boston, 2000.

[13] R. B. Vinter and H. Zheng. Necessary Conditions for Optimal Control Problem with State Constraints. Trans. Amer. Math. Soc. 350, 1998, pp.1181-1204.

[14] R. B. Vinter and G. Pappas. A Maximum Principle for Nonsmooth Optimal Control Problems with State Constraints. J. Math. Anal. Appl.89, 1982 pp. 212-232. 